\title{
Erratum: Conditions for coherence transformations under incoherent operations [Phys. Rev. A 91, 052120 (2015)]
}

Shuanping Du, Z Zhaofang Bai, and Yu Guo

(Received 18 January 2017; published 15 February 2017)

DOI: 10.1103/PhysRevA.95.029901

There is an error in the proof of the necessity of Theorem 1. That is, the cross term of the second equation of (21) does not vanish, which is due to $\delta_{j, i(l)}$ being dependent on $n$. Chitambar and Gour have made this point in [1]. In the following, we correct the error.

Now, we suppose $\operatorname{dim} H=3$ and there is an incoherent operation $\Phi$ that transforms $|\psi\rangle\langle\psi|$ to $|\phi\rangle\langle\phi|$. Let

$$
\Phi(|\psi\rangle\langle\psi|)=\sum_{n} K_{n}|\psi\rangle\left\langle\psi\left|K_{n}^{\dagger}=\right| \phi\right\rangle\langle\phi| .
$$

Hence there exist complex numbers $\alpha_{n}$ such that $K_{n}|\psi\rangle=\alpha_{n}|\phi\rangle$. Let $k_{j}^{(n)}(j=1,2,3)$ be the nonzero element of $K_{n}$ at the $j$ th column (if there is no nonzero element in the $j$ th column, then $\left.k_{j}^{(n)}=0\right)$. Suppose $k_{j}^{(n)}$ locates the $f_{n}(j)$ th row. Here, $f_{n}(j)$ is a function that maps $\{2,3\}$ to $\{1,2,3\}$ with the property that $1 \leqslant f_{n}(j) \leqslant j$. Let

$$
\delta_{s, t}=\left\{\begin{array}{ll}
1, & s=t \\
0, & s \neq t
\end{array} .\right.
$$

Then there is a permutation $\pi_{n}$ such that

$$
K_{n}=P_{\pi_{n}}\left(\begin{array}{ccc}
k_{1}^{(n)} & \delta_{1, f_{n}(2)} k_{2}^{(n)} & \delta_{1, f_{n}(3)} k_{3}^{(n)} \\
0 & \delta_{2, f_{n}(2)} k_{2}^{(n)} & \delta_{2, f_{n}(3)} k_{3}^{(n)} \\
0 & 0 & \delta_{3, f_{n}(3)} k_{3}^{(n)}
\end{array}\right) .
$$

From $\sum_{n} K_{n}^{\dagger} K_{n}=I$, we get that

$$
\begin{aligned}
& \sum_{n}\left|k_{j}^{(n)}\right|^{2}=1 \quad(j=1,2,3), \\
& \sum_{n} \overline{k_{1}^{(n)}} \delta_{1, f_{n}(2)} k_{2}^{(n)}=0, \\
& \sum_{n} \overline{k_{1}^{(n)}} \delta_{1, f_{n}(3)} k_{3}^{(n)}=0, \\
& \sum_{n}\left(\delta_{1, f_{n}(2)} \delta_{1, f_{n}(3)}+\delta_{2, f_{n}(2)} \delta_{2, f_{n}(3)}\right) \overline{k_{2}^{(n)}} k_{3}^{(n)}=0 .
\end{aligned}
$$

For $|\psi\rangle=\left(\psi_{1}, \psi_{2}, \psi_{3}\right)^{t}$, by a direct computation, one can get

$$
K_{n}|\psi\rangle=P_{\pi_{n}}\left(\begin{array}{c}
k_{1}^{(n)} \psi_{1}+\delta_{1, f_{n}(2)} k_{2}^{(n)} \psi_{2}+\delta_{1, f_{n}(3)} k_{3}^{(n)} \psi_{3} \\
\delta_{2, f_{n}(2)} k_{2}^{(n)} \psi_{2}+\delta_{2, f_{n}(3)} k_{3}^{(n)} \psi_{3} \\
\delta_{3, f_{n}(3)} k_{3}^{(n)} \psi_{3}
\end{array}\right)
$$

and so

$$
\begin{aligned}
& k_{1}^{(n)} \psi_{1}+\delta_{1, f_{n}(2)} k_{2}^{(n)} \psi_{2}+\delta_{1, f_{n}(3)} k_{3}^{(n)} \psi_{3}=\alpha_{n} \phi_{\pi_{n}^{-1}(1)}, \\
& \delta_{2, f_{n}(2)} k_{2}^{(n)} \psi_{2}+\delta_{2, f_{n}(3)} k_{3}^{(n)} \psi_{3}=\alpha_{n} \phi_{\pi_{n}^{-1}(2)}, \\
& \delta_{3, f_{n}(3)} k_{3}^{(n)} \psi_{3}=\alpha_{n} \phi_{\pi_{n}^{-1}(3)} .
\end{aligned}
$$


Applying $\sum_{n}|\cdot|^{2}$ to the above equations, we have

$$
\begin{aligned}
& \psi_{1}^{2}+\sum_{n} \delta_{1, f_{n}(2)}\left|k_{2}^{(n)}\right|^{2} \psi_{2}^{2}+\sum_{n} \delta_{1, f_{n}(3)}\left|k_{3}^{(n)}\right|^{2} \psi_{3}^{2}+\sum_{n} \delta_{1, f_{n}(2)} \delta_{1, f_{n}(3)} \overline{k_{2}^{(n)}} k_{3}^{(n)} \psi_{2} \psi_{3}+\sum_{n} \delta_{1, f_{n}(2)} \delta_{1, f_{n}(3)} \overline{k_{3}^{(n)}} k_{2}^{(n)} \psi_{3} \psi_{2} \\
& \quad=\sum_{n}\left|\alpha_{n}\right|^{2} \phi_{\pi_{n}^{-1}(1)}^{2}, \\
& \sum_{n} \delta_{2, f_{n}(2)}\left|k_{2}^{(n)}\right|^{2} \psi_{2}^{2}+\sum_{n} \delta_{2, f_{n}(3)}\left|k_{3}^{(n)}\right|^{2} \psi_{3}^{2}+\sum_{n} \delta_{2, f_{n}(2)} \delta_{2, f_{n}(3)} \overline{k_{2}^{(n)}} k_{3}^{(n)} \psi_{2} \psi_{3}+\sum_{n} \delta_{2, f_{n}(2)} \delta_{2, f_{n}(3)} \overline{k_{3}^{(n)}} k_{2}^{(n)} \psi_{3} \psi_{2} \\
& \quad=\sum_{n}\left|\alpha_{n}\right|^{2} \phi_{\pi_{n}^{-1}(2)}^{2}, \\
& \sum_{n} \delta_{3, f_{n}(3)}\left|k_{3}^{(n)}\right|^{2} \psi_{3}^{2}=\sum_{n}\left|\alpha_{n}\right|^{2} \phi_{\pi_{n}^{-1}(3)}^{2} .
\end{aligned}
$$

Note that, for $s=1,2,3$,

$$
\sum_{n}\left|\alpha_{n}\right|^{2} \phi_{\pi_{n}^{-1}(s)}^{2}=\sum_{n, \pi_{n}^{-1}(s)=1}\left|\alpha_{n}\right|^{2} \phi_{1}^{2}+\sum_{n, \pi_{n}^{-1}(s)=2}\left|\alpha_{n}\right|^{2} \phi_{2}^{2}+\sum_{n, \pi_{n}^{-1}(s)=3}\left|\alpha_{n}\right|^{2} \phi_{3}^{2} .
$$

Let $d_{i j}=\sum_{n, \pi_{n}^{-1}(i)=j}\left|\alpha_{n}\right|^{2}, 1 \leqslant i, j \leqslant 3$, then the matrix $D=\left(d_{i j}\right)$ is a doubly stochastic matrix, since $\sum_{n}\left|\alpha_{n}\right|^{2}=1$. Furthermore,

$$
D\left(\phi_{1}^{2}, \phi_{2}^{2}, \phi_{3}^{2}\right)^{t}=\left(\sum_{n}\left|\alpha_{n}\right|^{2} \phi_{\pi_{n}^{-1}(1)}^{2}, \sum_{n}\left|\alpha_{n}\right|^{2} \phi_{\pi_{n}^{-1}(2)}^{2}, \sum_{n}\left|\alpha_{n}\right|^{2} \phi_{\pi_{n}^{-1}(3)}^{2}\right)^{t} .
$$

This implies that

$$
\left(\sum_{n}\left|\alpha_{n}\right|^{2} \phi_{\pi_{n}^{-1}(1)}^{2}, \sum_{n}\left|\alpha_{n}\right|^{2} \phi_{\pi_{n}^{-1}(2)}^{2}, \sum_{n}\left|\alpha_{n}\right|^{2} \phi_{\pi_{n}^{-1}(3)}^{2}\right)^{t} \prec\left(\phi_{1}^{2}, \phi_{2}^{2}, \phi_{3}^{2}\right)^{t} .
$$

On the other hand, note that in the first equation of (6),

$$
\begin{aligned}
& \sum_{n} \delta_{1, f_{n}(2)}\left|k_{2}^{(n)}\right|^{2} \psi_{2}^{2}+\sum_{n} \delta_{1, f_{n}(3)}\left|k_{3}^{(n)}\right|^{2} \psi_{3}^{2}+\sum_{n} \delta_{1, f_{n}(2)} \delta_{1, f_{n}(3)} \overline{k_{2}^{(n)}} k_{3}^{(n)} \psi_{2} \psi_{3}+\sum_{n} \delta_{1, f_{n}(2)} \delta_{1, f_{n}(3)} \overline{k_{3}^{(n)}} k_{2}^{(n)} \psi_{3} \psi_{2} \\
& =\sum_{n}\left|\delta_{1, f_{n}(2)} k_{2}^{(n)} \psi_{2}+\delta_{1, f_{n}(3)} k_{3}^{(n)} \psi_{3}\right|^{2} .
\end{aligned}
$$

This tells us that

$$
\psi_{1}^{2} \leqslant \sum_{n}\left|\alpha_{n}\right|^{2} \phi_{\pi_{n}^{-1}(1)}^{2}
$$

Adding the first and the second equations of (6) together, by (3), we have

$$
\psi_{1}^{2}+\psi_{2}^{2} \leqslant \sum_{n}\left|\alpha_{n}\right|^{2} \phi_{\pi_{n}^{-1}(1)}^{2}+\sum_{n}\left|\alpha_{n}\right|^{2} \phi_{\pi_{n}^{-1}(2)}^{2} .
$$

From the definition of majorization, one can check that

$$
\left(\psi_{1}^{2}, \psi_{2}^{2}, \psi_{3}^{2}\right)^{t} \prec\left(\sum_{n}\left|\alpha_{n}\right|^{2} \phi_{\pi_{n}^{-1}(1)}^{2}, \sum_{n}\left|\alpha_{n}\right|^{2} \phi_{\pi_{n}^{-1}(2)}^{2}, \sum_{n}\left|\alpha_{n}\right|^{2} \phi_{\pi_{n}^{-1}(3)}^{2}\right)^{t} .
$$

Combining (9) with (11), we have

$$
\left(\psi_{1}^{2}, \psi_{2}^{2}, \psi_{3}^{2}\right)^{t} \prec\left(\phi_{1}^{2}, \phi_{2}^{2}, \phi_{3}^{2}\right)^{t}
$$

The research was supported by the National Science Foundation of China (11671332).

[1] E. Chitambar and G. Gour, Phys. Rev. A 94, 052336 (2016). 